\title{
PAPER
}

\section{Neurophysiological modulation of the subthalamic nucleus by pallidal stimulation in Parkinson's disease}

\section{Sterio, A Rezai, A Mogilner, M Zonenshayn, J-M Gracies, K Kathirithamby, A Berić}

See end of article for

authors' affiliations

J Neurol Neurosurg Psychiatry 2002;72:325-328

Correspondence to:

Dr A Berić, Department of

Neurology, Hospital for

Joint Diseases, 301 East

17th Street, New York, NY

10003, USA;

Received 11 July 2001

In revised form

30 October 2001

Accepted

5 November 2001

\begin{abstract}
Objectives: Current models of basal ganglia dysfunction in Parkinson's disease suggest a pivotal role of subthalamic nucleus (STN) hyperactivity. There is a direct excitatory output to the globus pallidus internus (GPi), which in turn hyperinhibits the motor thalamus and leads to a lack of cortical facilitation. The model, however, does not address the reciprocal influence of GPi on STN activity.

Methods: Measurement of immediate changes in STN single cell activity after GPi deep brain stimulation (DBS).

Results: An opposite effect of GPi DBS in the dorsal versus ventral STN was found. There was an almost exclusive reduction of firing rate in the dorsal region of the STN, whereas the cells in the ventral region exhibited facilitation similar to the recordings from the substantia nigra pars reticulata.

Conclusion: Although these findings require confirmation, they suggest that the current theories of GPi DBS action, which do not include a GPi-STN modulation, are most likely incomplete.
\end{abstract}

C urrent models of basal ganglia organisation in Parkinson's disease suggest that the subthalamic nucleus (STN) exerts a facilitatory influence on the globus pallidus internus (Gpi). ${ }^{1-6}$ As this output increases, the resulting hyperactivity of GPi leads to excessive thalamic inhibition and consequent cortical hypoactivity, which accounts for the typical clinical findings of bradykinesia and akinesia. Besides existing controversy regarding the level of activity in the globus pallidus externus (GPe) and its role in STN hyperactivity, ${ }^{7}$ little is known about other pallidal efferent pathways, and their role in the normal and parkinsonian brain. Despite the lack of evidence of direct pathways interconnecting all basal ganglia structures, it is thought that there are functional feedback systems at every structural/functional level.

Based on the current basal ganglia model, however, it is unclear if and how changes in GPi activity influence STN hyperactivity in Parkinson's disease. We had the opportunity to study this interaction in a patient with Parkinson's disease with implanted pallidal stimulators undergoing bilateral STN deep brain stimulator (DBS) implantation. We recorded single cell activity from the STN during and immediately after pallidal stimulation and analyzed the changes induced by GPi stimulation in different regions of the STN and SNr.

\section{MATERIAL AND METHODS}

The patient was a 68 year old man with a 27 year history of idiopathic Parkinson's disease. In 1994, as the medication treatment alone no longer controlled his symptoms, the patient underwent implantation of bilateral monopolar GPi stimulators (Model 3388, Medtronic, Minneapolis, MN, USA) with good initial clinical benefit. However, in the ensuing years he required increasingly frequent changes of the pulse generators (eventually every 5 months) due to increasing current requirements. Furthermore, although his tremor and rigidity were much improved with stimulation, his gait had deteriorated over time and had not responded to GPi DBS. He was referred to our centre for bilateral STN DBS implantation. Antiparkinsonian medications were withheld the night before surgery while stimulation was continued up until, and during most surgery. After Leksell frame (Model G, Elekta, Atlanta, GA, USA) application, the MRI localiser was attached to the frame and the patient underwent MR and CT imaging. Axial
CT images ( $1 \mathrm{~mm}$ ) were obtained. One to $3 \mathrm{~mm}$ axial, coronal, and sagittal MR images were obtained coplanar to a line connecting the anterior commissure (AC) to the posterior commissure (PC). The imaging data was transferred from the CT and MR scanners to the Compass (Compass International, Rochester, MN, USA) stereotactic system via a computer network. The system is equipped with a digitised stereotactic atlas that can be reformatted to an individual patient's AC and PC and overlaid onto the appropriate MR imaging section.

The stereotactic $\mathrm{x}, \mathrm{y}$, and $\mathrm{z}$ coordinates of the STN relative to the AC and PC were determined on axial and sagittal Tl weighted MRI according to the technique described previously by Zonenshayn et al. ${ }^{8}$ Briefly, two indirect methods were concomitantly used. For indirect midcommissural based targeting a point $4 \mathrm{~mm}$ posterior, $5 \mathrm{~mm}$ inferior, and $13 \mathrm{~mm}$ lateral to the midcommissural point was chosen. For atlas based targeting, the appropriate parasagittal atlas sections from Schaltenbrand and Wahren ${ }^{9}$ and Morel et al ${ }^{10}$ were employed after they had been scaled, rotated, and translated with respect to the individual patient's intercommissural line. A typical laterality of 12.5-13 mm from midline was used, thereby maximising the anterior-posterior diameter of the STN. Our anatomical target was chosen at the centre of the STN on a $13 \mathrm{~mm}$ reformatted parasagittal Schaltenbrand and Wahren' brain map, or the ventral-posterior border of the STN on a $12.6 \mathrm{~mm}$ parasagittal atlas section from Morel et al. ${ }^{10}$ Direct MR targeting could not be performed due to the presence of significant artifact emanating from the monopolar GPi electrodes, which obscured the view of the STN. In addition, CT imaging was used to confirm the AC and PC locations, as well as the location of pallidal DBS electrodes.

After completing the preoperative imaging studies bilateral 4-6 cm linear skin incisions were performed. Bilateral $14 \mathrm{~mm}$ diameter burr holes were then created anterior to the coronal suture and $2.5 \mathrm{~cm}$ lateral to midline under local anaesthesia and mild sedation with diprivan. A $1.1 \mathrm{~mm}$ diameter impedance monitoring probe (Radionics, Burlington, MA,

Abbreviations: STN, subthalamic nucleus; GPi, globus pallidus internus; GPe, globus pallidus externus; DBS, deep brain stimulator; AC, anterior commissure; PC, posterior commissure; ISI, interspike interval 
Table 1 STN and SNr cells in a patient with bilateral pallidal DBS

\begin{tabular}{llll}
\hline & $\begin{array}{l}\text { Total cells } \\
\text { recorded }\end{array}$ & $\begin{array}{l}\text { Mean firing } \\
\text { rate }(\mathrm{Hz})\end{array}$ & $\begin{array}{l}\text { Cells with } \\
\text { GPi DBS }\end{array}$ \\
\hline STN: & 32 & 49 & 11 \\
$\quad$ Right side & 26 & 41 & 6 \\
$\quad$ Left side & 14 & 82 & 2 \\
SNr: & 9 & 90 & 1 \\
$\quad$ Right side & & & \\
Left side & & & \\
\hline
\end{tabular}

USA) was used to create a tract for the subsequent microelectrode cannula to a point $15 \mathrm{~mm}$ superficial to the STN target. At this time, physiological localisation commenced.

Recordings were obtained using a technique previously described. ${ }^{11}{ }^{12}$ The exposed tungsten microelectrode tip length was $15-40 \mu$, with a typical impedance of 0.5 to $1.0 \mathrm{MOhm}$ at $1000 \mathrm{~Hz}$. A digital hydraulic microdrive was employed to advance the microelectrode in sub-mm steps beyond the most distal aspect of the $1.1 \mathrm{~mm}$ diameter cannula into the brain parenchyma (beginning $15 \mathrm{~mm}$ superficial to the selected anatomical STN target). The electrical signal derived from the electrode was filtered (300 to $10000 \mathrm{~Hz}$ ) and amplified (10 $000 \times$ ) by a DAM-80 amplifier (WPI, Sarasota, Fl, USA), and displayed on a Tektronix 2214 oscilloscope (Tektronix Inc, Beaverton, OR, USA), while being fed to a Grass audiomonitor (Grass Instruments, Quincy, MA, USA). In addition, for each encountered cell, spontaneous activity was digitised and stored on a magnetic tape. Detailed off line analysis was later accomplished using Spike 2 software (Cambridge Instruments, Cambridge, UK). Mean rates of discharge were calculated for each cell that was clearly distinguishable from background activity. Interspike interval (ISI) histograms were constructed for each isolated neuron, employing a time base of $200 \mathrm{~ms}$ and bin width of $0.5 \mathrm{~ms}$. After obtaining a stable single cell baseline recording, the pallidal stimulator ipsilateral to the recorded STN was activated for 1 minute with an intensity of $4.5 \mathrm{~V}$, frequency of $185 \mathrm{~Hz}$, and pulse duration of $450 \mu \mathrm{s}$. These parameters yielded maximal clinical benefit preoperatively. The cellular activity was recorded for 1 minute after the discontinuation of stimulation. During the actual periods of pallidal stimulation STN cell discharges could not be recorded due to a large stimulus artifact. Repeated trials were recorded and analyzed. The same procedure was performed for cells in both the dorsal and ventral portions of the STN, as well as for cells within the dorsal portion of the SNr.

\section{RESULTS}

The posterior ventral portion of the right STN was physiologically localised to a point $12.5 \mathrm{~mm}$ lateral, $4.1 \mathrm{~mm}$ posterior,

\section{Before

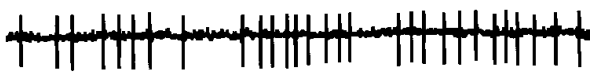

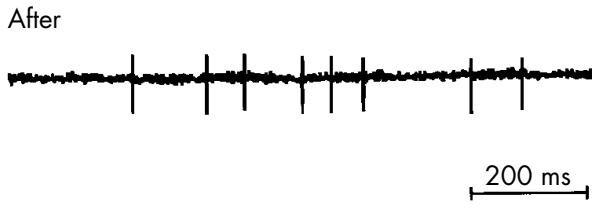

Figure 1 Dorsal STN cell firing before and the first second immediately after GPi stimulation in a patient with implanted bilateral GPi stimulators undergoing bilateral STN DBS surgery. Note a significant decrease in firing rate after the pallidal stimulation.

and $4.7 \mathrm{~mm}$ ventral to the midcommissural point. Similarly, the left STN target was physiologically localised to a point 11.5 $\mathrm{mm}$ lateral, $3.1 \mathrm{~mm}$ posterior, and $4.7 \mathrm{~mm}$ ventral to the midcommissural point. Based on preoperative CT imaging, the right sided pallidal electrode was $17 \mathrm{~mm}$ lateral, $0.5 \mathrm{~mm}$ posterior, and $8 \mathrm{~mm}$ ventral to the midcommissural point. In turn, the left sided pallidal electrode was $17 \mathrm{~mm}$ lateral, $1 \mathrm{~mm}$ posterior, and $3 \mathrm{~mm}$ ventral to the midcommissural point.

While performing microelectrode recordings from within the STN, intraoperative stimulation of the ipsilateral GPi via the previously implanted monopolar DBS electrodes demonstrated a change in STN firing frequency. From a total of 58 encountered cells within the STN, 17 random stable STN cells were recorded before and immediately after GPi stimulation (table 1). Ipsilateral GPi stimulation resulted in changes of the baseline discharges in all of the recorded cells (table 2). The direction of change varied along the recording axis of the STN. In the dorsal half of the STN, eight of 11 recorded cells demonstrated inhibition with GPi stimulation, with a decrease in the mean firing frequency (figs 1 and 2). In the ventral STN, the opposite effect was noted: five of six recorded cells showed an increase in firing rate with GPi stimulation (fig 3). The distribution of the cell behaviour along the recording axis through the STN and the SNr is shown in figure 4. Due to this diametrically opposite behaviour the overall mean STN firing rate did not demonstrate any change with the GPi DBS. During continued recording of a given cell there was a slow but definite return of cell discharges to baseline prestimulation levels. The average time for return to the baseline was $25 \mathrm{sec}-$ onds. Contralateral pallidal stimulation was performed while recording from a small number of both dorsal and ventral STN cells, with no significant changes in the firing frequency.

The average firing rate of all dorsal STN cells was $47.6 \mathrm{~Hz}$. The subset of cells that were seen during and after GPi stimulation had a prestimulation firing rate of $49.5 \mathrm{~Hz}$. The average

Table 2 Single cell firing rates $(\mathrm{Hz})$ in dorsal and ventral regions of the STN, and dorsal SNr before and immediately after GPi DBS

\begin{tabular}{|c|c|c|c|c|c|c|}
\hline & Cell 1 & Cell 2 & Cell 3 & Cell 4 & Cell 5 & Cell 6 \\
\hline \multicolumn{7}{|c|}{ Dorsal STN right side: } \\
\hline Before & 30 & 42 & 61 & 57 & 55 & \\
\hline After & 20 & 23 & 29 & 26 & 37 & \\
\hline \multicolumn{7}{|c|}{ Dorsal STN left side: } \\
\hline Before & 48 & 17 & 40 & 20 & 90 & 84 \\
\hline After & 30 & 7 & 75 & 8 & 128 & 106 \\
\hline \multicolumn{7}{|c|}{ Ventral STN right side: } \\
\hline Before & 84 & 66 & 55 & 66 & 66 & 33 \\
\hline After & 100 & 118 & 79 & 112 & 84 & 20 \\
\hline \multicolumn{7}{|l|}{ SNr: } \\
\hline Before & 25 & 84 & 96 & & & \\
\hline After & 60 & 112 & 120 & & & \\
\hline
\end{tabular}

Bold indicates an exception to the orientation of the firing rate changes. 

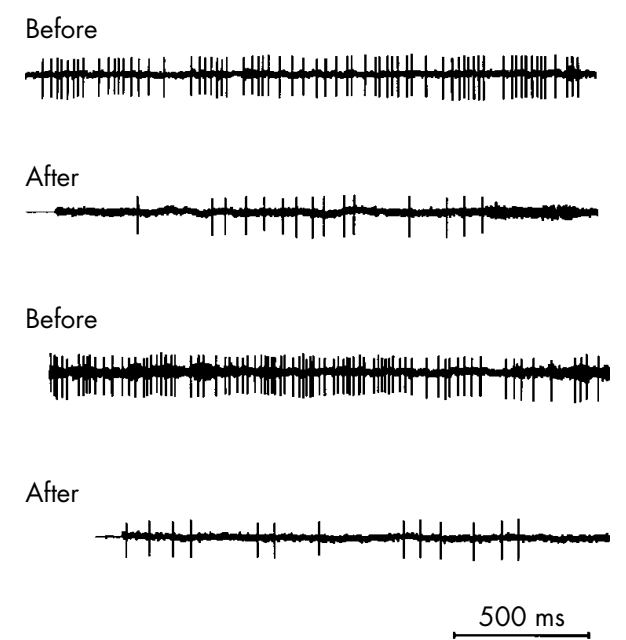

Figure 2 Different dorsal STN cell firing before and the first second immediately after GPi stimulation (upper traces). Note also a significant decrease in firing rate of the same cell after repeated (lower traces) trials of pallidal stimulation.

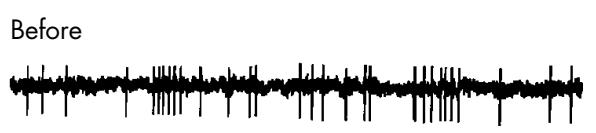

After

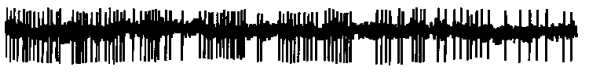

$200 \mathrm{~ms}$

Figure 3 Ventral STN cell firing before and immediately after GPi stimulation in the same patient. Note a significant increase in firing rate after the pallidal stimulation.

firing rate for the ventral cells was $58.4 \mathrm{~Hz}$, while the subset found had a baseline firing rate of $61.7 \mathrm{~Hz}$. Average STN cell density was 5.1 cells $/ \mathrm{mm}$ without any significant left-right differences.

The regularly firing SNr was encountered after exiting from the irregularly firing ventral STN and traversing a $1-2 \mathrm{~mm}$ acellular zone. From a total of $23 \mathrm{SNr}$ cells, three random cells were recorded before and immediately after the ipsilateral GPi stimulation. All SNr cells demonstrated increased firing rates with GPi stimulation (table 2).

\section{DISCUSSION}

Microelectrode recordings in the STN demonstrated a remarkable modulation of cellular activity with pallidal stimulation. The effects were transient, with a fast return to baseline after pallidal stimulation had ceased. Due to a considerable stimulus artifact it was not possible to record STN activity during the actual onset of GPi stimulation. Therefore, the latency of the STN response is unknown. Based on prior studies concluding that there are no direct axonal pathways from the GPi to the STN, ${ }^{6}{ }^{13}$ the effect is either polysynaptic or a retrograde depolarisation blockade of glutaminergic STN projection fibres. These findings also suggest that pallidal DBS stimulation is capable of both inhibiting and facilitating STN cellular activity. It seems that a high frequency GPi stimulation leads to a decrease in cellular firing rates within the dorsal portion of STN, but a further increase within the ventral portion of the STN and SNr. Simple

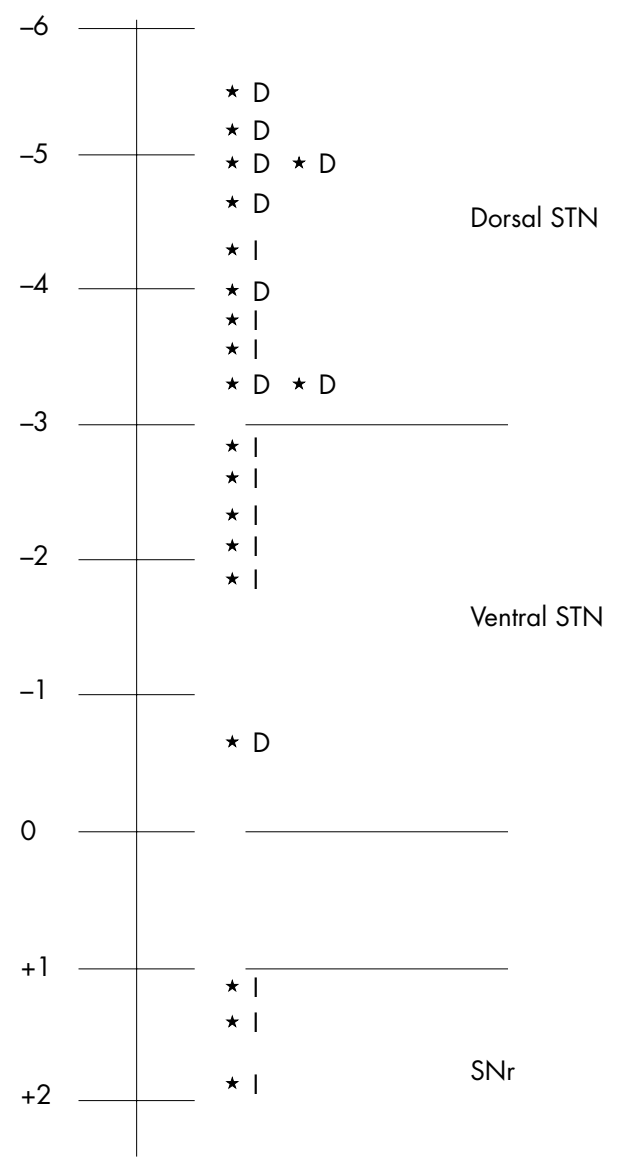

Figure 4 A presumed trajectory through the STN nucleus with both right and left STN cells combined. D=decrease in firing rate after GPi stimulation. I=increase in firing rate after GPi stimulation. Note a peculiar distribution of the decreasing firing rate cells in the upper half of the STN and increasing firing rate cells in the middle lower portion of the STN.

retrograde depolarisation of the efferent STN axons (STN-GPi pathway) would not lead to this differential response. Interestingly, in another study of 26 patients with Parkinson's disease undergoing STN DBS surgery, a similar disparity in basal firing rate was found between the dorsal and ventral halves of the STN. ${ }^{12}$ Our findings are consistent with a rostrocaudal organisation and segregation of the dorsal lateral to ventral-medial STN. ${ }^{13-18}$ The results demonstrate a rather efficient functional feedback system between the GPi and the STN that may be taken into account in the further development of basal ganglia models in the normal and parkinsonian states.

It could be argued that perhaps the pallidal DBS electrode was stimulating GPe instead of GPi, and thus STN activity was modulated by anterograde GPe inhibitory projections to the STN, as predicted in the current basal ganglia model. This is unlikely, however, as the coordinates of the pallidal electrodes corresponded with the location of GPi. In addition, unlike the typical Medtronic quadripolar electrodes currently employed (models 3387and 3389), our patient had an implanted monopolar system which uses only one active contact site and makes it easier to determine its position. Furthermore, the patient had a persistent positive effect on tremor, rigidity, and dyskinesias for more than 5 years after implantation.

It could be argued that cells in the lower half of the STN are in fact $\mathrm{SNr}$ cells as they exhibited a similar pattern of increase in firing rate with GPi stimulation. Interestingly, the encountered cells were in the central ventral area of the STN and not towards the lower part of the STN (fig 4). In addition, the cells 
encountered after the 1-2 $\mathrm{mm}$ acellular zone correspond to the anatomical location of the $\mathrm{SNr}$ with respect to the coordinates of the AC and PC. Therefore, it seems that the cluster of cells with an increased firing rate as a result of GPi stimulation is located within the body of the STN.

The firing rates of the STN cells, in general, were higher than is typically reported, and were even higher than our own data. ${ }^{12}$ The average firing rate of our patients is $47 \mathrm{~Hz}$, with a dorsal STN rate of $52 \mathrm{~Hz}$ and a ventral rate of $43 \mathrm{~Hz}$. The firing rates in this study showed an opposite effect, the baseline dorsal firing rates are lower than the ventral firing rates. The difference is relatively minor for the dorsal STN $(52 \mathrm{~Hz} v 48 \mathrm{~Hz})$, while the ventral rates are more disparate $(43 \mathrm{~Hz} v 58 . \mathrm{Hz})$. As GPi stimulation showed a tendency to decrease the dorsal firing rate and increase the ventral firing rate, we could speculate that the baseline firing rates are the result of relatively short discontinuation of GPi stimulation and, therefore, they reflect some carryover GPi stimulation effect.

We elected to implant bilateral STN stimulators due to progressive axial symptoms. In addition, the monopolar system had been modified to permit a connection to the Itrel II HF system, which typically drives a quadripolar DBS electrode. Due to these modifications and the resultant higher current demands, we debated whether to remove the still beneficial pallidal electrodes and replace them with a newer quadripolar design. However, the new position after removal might not have been equally effective. As the patient relied on chronic stimulation for control of his Parkinson's disease we elected to implant the STN stimulators and keep the pallidal electrodes in place until the proper STN settings could be obtained. At this point, the pallidal stimulators would be switched off and kept in reserve in case of any STN DBS malfunction.

Mild sedation with diprivan during surgery should not interfere with the microelectrode recording, although it may decrease the overall firing frequency. However, the frequency of baseline STN discharges was comparable with the other patients undergoing STN DBS implantation who were kept fully awake. ${ }^{12}$ Additionally, we found changes in discharge pattern during pallidal stimulation using the same cells as a control.

Although the stimulation periods were brief, and thus any inference regarding the effects of chronic stimulation is speculative, there is no reason to think that the effects of GPi stimulation differ between acute and chronic conditions. Based on these results, it may not be surprising that recordings performed in patients with prior pallidotomy during STN DBS surgery demonstrated reduced STN cell firing rates in the dorsolateral sensorimotor division of the nucleus. ${ }^{19}$ Interestingly, but perhaps not surprisingly, the cell discharge frequencies in our patient off GPi stimulation were on both sides similar to "non-pallidotomised" patients with Parkinson's disease undergoing STN DBS implantation. ${ }^{12}$

Although these findings require confirmation, they suggest a new additional mechanism of GPi DBS action in Parkinson's disease. In addition, the currently accepted theories of functional connectivity of the basal ganglia might benefit from discussing a potential feed forward GPi-STN interaction and the more controversial STN segregation. ${ }^{7120}$
Competing interests: JMG has been an investigator in a Medtronic sponsored trial. All other authors, no competing interests declared.

\section{Authors' affiliations}

D Sterio, A Rezai, A Mogilner, M Zonenshayn, Department of Neurosurgery, NYU School of Medicine, NY, USA

A Berić, Department of Neurology, NYU School of Medicine, NY, USA

J-M Gracies, Department of Neurology, Mount Sinai School of

Medicine, NY, USA

M Zonenshayn, Department of Neurosurgery, Weill Medical College of Cornell University, NY, USA

K Kathirithamby, Department of Anesthesiology, Hospital for Joint

Diseases, New York, NY, USA

\section{REFERENCES}

1 Albin RL, Young AB, Penney JB. The functional anatomy of basal ganglia disorders. Trends Neurosci 1989;12:366-75.

2 Alexander GE, Crutcher MD. Functional architecture of basal ganglia circuits: neural substrates of parallel processing. Trends Neurosci 1990;13:266-71

3 DeLong MR. Primate models of movement disorders of basal ganglia origin. Trends Neurosci 1990;13:281-5.

4 Wichman T, DeLong MR. Pathophysiology of parkinsonian motor abnormalities. Adv Neurol 1993;60:53-61.

5 Marsden CD, Obeso JA. The function of the basal ganglia and the paradox of stereotaxic surgery in Parkinson's disease. Brain 1994:117:877-97.

6 Smith $Y$, Bevan MD, Shink E, et al. Microcircuitry of the direct and indirect pathways of the basal ganglia. Neuroscience 1998;86:353-87.

7 Levy R, Hazrati L-N, Herrero M-T, et al. Re-evaluation of the functional anatomy of the basal ganglia in normal and Parkinsonian states. Neuroscience 1997;76:335-43.

8 Zonenshayn M, Rezai AR, Mogilner AY, et al. Comparison of anatomic and neurophysiological methods for subthalamic nucleus targeting. Neurosurgery 2000;47:282-94.

9 Schaltenbrand G, Wahren W. Atlas for stereotaxy of the human brain. Stuttgart: Georg Thieme, 1977

10 Morel A, Magnin M, Jeanmonod D. Multiarchitectonic and stereotactic atlas of the human thalamus. J Comp Neurol 1997;387:588-630.

11 Sterio D, Beric A, Dogali M, et al. Neurophysiological properties of pallidal neurons in Parkinson's disease. Ann Neurol 1994;35:586-91.

12 Sterio D, Zonenshayn M, Mogilner A, et al. Neurophysiologic refinement of subthalamic nucleus targeting. Neurosurgery 2001 (in press).

13 Carpenter MB, Carleton SC, Keller JT, et al. Connections of the subthalamic nucleus in the monkey. Brain Res 1981;224:1-29.

14 Parent A, Smith Y. Organization of efferent projections of the subthalamic nucleus in the squirrel monkey as revealed by retrograde labeling methods. Brain Res 1987;436:296-310.

15 Parent A, Smith Y, Filion M, et al. Distinct afferents to internal and external pallidal segments in the squirrel monkey. Neurosci Lett 1989;96:140-4.

16 Smith Y, Bolam JP, Krosigk M. Topographical and synaptic organization of the GABA-containing pallidosubthalamic projection in the rat. Eur $J$ Neurosci 1990;2:500-11

17 Parent A, Hazrati LN. Functional anatomy of the basal ganglia 2. The place of subthalamic nucleus and external pallidum in basal ganglia circuitry. Brain Res Rev 1995;20: 128-54.

18 Hardman CD, Halliday GH, McRitchie DA, et al. The subthalamic nucleus in Parkinson's disease and progressive supranuclear palsy. $J$ Neuropathol Exp Neurol 1997;56:132-42.

19 Mogilner AY, Rezai AR, Zonenshayn M, et al. Neurophysiological characteristics of the subthalamic nucleus in patients with prior pallidotomy. J Neurosurg 2001 (in press).

20 Wichman T, DeLong MR. Models of basal ganglia function and pathophysiology of movement disorders. Neurosurg Clin N Am 1998:9:223-36. 